\title{
Population-based input function for TSPO quantification and kinetic modeling with $\left[{ }^{11} \mathrm{C}\right]-\mathrm{DPA}-713$
}

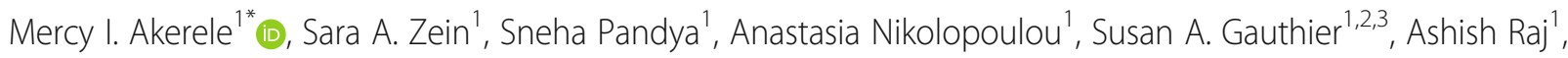
Claire Henchcliffe ${ }^{1,2}$, P. David Mozley ${ }^{1}$, Nicolas A. Karakatsanis' ${ }^{1}$ Ajay Gupta ${ }^{1}$, John Babich ${ }^{1}$ and Sadek A. Nehmeh ${ }^{1}$

\author{
* Correspondence: mia4006@med. \\ cornell.edu \\ ${ }^{1}$ Department of Radiology, Weill \\ Cornell Medical College, New York, \\ NY 10021, USA \\ Full list of author information is \\ available at the end of the article
}

\begin{abstract}
Introduction: Quantitative positron emission tomography (PET) studies of neurodegenerative diseases typically require the measurement of arterial input functions (AIF), an invasive and risky procedure. This study aims to assess the reproducibility of $\left[{ }^{11} \mathrm{C}\right] \mathrm{DPA}-713$ PET kinetic analysis using population-based input function (PBIF). The final goal is to possibly eliminate the need for AIF.

Materials and methods: Eighteen subjects including six healthy volunteers (HV) and twelve Parkinson disease (PD) subjects from two $\left[{ }^{11} \mathrm{C}\right]-D P A-713$ PET studies were included. Each subject underwent 90 min of dynamic PET imaging. Five healthy volunteers underwent a test-retest scan within the same day to assess the repeatability of the kinetic parameters. Kinetic modeling was carried out using the Logan total volume of distribution $\left(V_{T}\right)$ model. For each data set, kinetic analysis was performed using a patient-specific AIF (PSAIF, ground-truth standard) and then repeated using the PBIF. PBIF was generated using the leave-one-out method for each subject from the remaining 17 subjects and after normalizing the PSAIFs by 3 techniques: (a) Weight subject $\times$ Dose $_{\text {Injected }}($ (b) area under AIF curve (AUC), and (c) Weight $_{\text {subject }} \times A \cup C$. The variability in the $V_{T}$ measured with PSAIF, in the test-retest study, was determined for selected brain regions (white matter, cerebellum, thalamus, caudate, putamen, pallidum, brainstem, hippocampus, and amygdala) using the Bland-Altman analysis and for each of the 3 normalization techniques. Similarly, for all subjects, the variabilities due to the use of PBIF were assessed.

Results: Bland-Altman analysis showed systematic bias between test and retest studies. The corresponding mean bias and 95\% limits of agreement (LOA) for the studied brain regions were $30 \%$ and $\pm 70 \%$.

Comparing PBIF- and PSAIF-based $V_{T}$ estimate for all subjects and all brain regions, a significant difference between the results generated by the three normalization techniques existed for all brain structures except for the brainstem ( $P$-value $=0.095$ ).

The mean \% difference and 95\% LOA is $-10 \%$ and $\pm 45 \%$ for

Weight $_{\text {subject }} \times$ Dose $_{\text {Injected; }}+8 \%$ and $\pm 50 \%$ for $A U C$; and $+2 \%$ and $\pm 38 \%$ for Weight $_{\text {subject }} \times A \cup C$. In all cases, normalizing by Weight subject $\times A \cup C$ yielded the smallest $\%$ bias and variability (\% bias $= \pm 2 \%$; LOA $= \pm 38 \%$ for all brain regions).

(Continued on next page)
\end{abstract}


(Continued from previous page)

Estimating the reproducibility of PBIF-kinetics to PSAIF based on disease groups (HV/ $P D)$ and genotype (MAB/HAB), the average $V_{T}$ values for all regions obtained from PBIF is insignificantly higher than PSAIF (\%difference $=4.53 \%, P$-value $=0.73$ for $\mathrm{HAB}$; and $\%$ difference $=0.73 \%, P$-value $=0.96$ for MAB). PBIF also tends to overestimate the difference between PD and HV for HAB (\% difference $=32.33 \%$ versus $13.28 \%$ ) and underestimate it in MAB (\%difference $=6.84 \%$ versus $20.92 \%$ ).

Conclusions: PSAIF kinetic results are reproducible with PBIF, with variability in $V_{T}$ within that obtained for the test-retest studies. Therefore, $V_{T}$ assessed using PBIFbased kinetic modeling is clinically feasible and can be an alternative to PSAIF.

Keywords: Population-based input function, Kinetic modeling, $\left[{ }^{11} \mathrm{C}\right] \mathrm{DPA}-713$, Normalization

\section{Introduction}

$18-\mathrm{kDa}$ translocator protein (TSPO) receptor has been shown as a potential target for imaging neuroinflammation using PK-11195 PET [1-3]. Recently, a putative antagonist of TSPO, $\left[{ }^{11} \mathrm{C}\right]-\mathrm{N}, \mathrm{N}$-diethyl-2[2-(4-methoxyphenyl)-5,7-dimethyl-pyrazolol[1,5- $\left.\alpha\right]$ pyrimidin-3-yl]-acetamide $\left(\left[{ }^{11} \mathrm{C}\right] \mathrm{DPA}-713\right)$, was developed concurrently with the TSPO agonist, fluoro-ethoxy derivative $\left[{ }^{18} \mathrm{~F}\right] \mathrm{DPA}-714$ [4-6]. Both $\left[{ }^{11} \mathrm{C}\right] \mathrm{DPA}-713$ and $\left[{ }^{18} \mathrm{~F}\right] \mathrm{DPA}-714$ were shown to have higher affinity than the first generation TSPO tracer $\left[{ }^{11} \mathrm{C}\right]-(\mathrm{R})-\mathrm{PK} 11195[4,7,8]$. Several studies have now demonstrated the usefulness of $\left[{ }^{11} \mathrm{C}\right] \mathrm{DPA}-713$ PET in quantifying neuroinflammation in different diseases, including multiple sclerosis (MS), Parkinson's disease (PD), and Alzheimer disease (AD), both in animal and human studies [4, 9-12].

In PET, kinetic modeling is often essential for the accurate quantification of tracer uptake and metabolism in the tissue. This often requires the measurement of the tracer concentration in the arterial blood over time. However, this practice is often limited in terms of its invasive nature and associated risks to the subjects, as well as risky blood sample handling [13]. The need for inserting arterial lines in patients leads to significant discomfort and patient burden. In practical clinical trial settings, this often proves a key workflow bottleneck and can also adversely influence subject cooperation and accrual [14].

An alternative technique such as an image-derived input function (IDIF) $[15,16]$ or population-based input function (PBIF) $[17,18]$ can facilitate the adoption of PET protocols requiring input functions. In brain studies, IDIF is usually deduced from the dynamic images of the carotid arteries and hence is susceptible to partial volume effect [15-17]. Previous studies showed the feasibility of PBIF as a robust alternative to IDIF for some radiopharmaceuticals $[17,18]$. PBIF is generated by averaging the normalized patient-specific arterial input functions (PSAIFs) deduced from a cohort of subjects. Several normalization techniques have been reported in the literature, for example, traditional scaling using blood samples by correlating the measured plasma activity with the AUC [17]; correlation of the PBIF with PSAIF venous samples [19]; scaling by injected dose and weight [20,21]; and non-invasive scaling using individual parameters like weight, body surface area (BSA), and lean body mass (LBM) [17]. Many studies have assessed the feasibility of PBIF for kinetic analysis using $\left[{ }^{18}\right.$ F]FDG [18, 22-25], yet very few studies involved neuroreceptor PET tracers $[17,26]$, including TSPO brain 
studies $[19,21,27]$. To the best of our knowledge, no PET kinetic modeling study has been performed with $\left[{ }^{11} \mathrm{C}\right] \mathrm{DPA}-713$ using PBIF.

The main aim of this study is to assess the feasibility of using PBIF instead of the patient-specific AIF for $\left[{ }^{11} \mathrm{C}\right] \mathrm{DPA}-713$ PET kinetic modeling. This was done by first estimating the test-retest repeatability of the $\left[{ }^{11} \mathrm{C}\right] \mathrm{DPA}-713$ PET imaging in healthy subjects. Based on the test-retest results, we then assessed the reproducibility of kinetic analysis of $\left[{ }^{11} \mathrm{C}\right] \mathrm{DPA}-713$ dynamic PET images of the brain with PBIF compared to PSAIF in healthy and PD subjects. The effect of PSAIF normalization techniques on the PBIF-based kinetic results was also investigated.

\section{Materials and methods}

\section{Subjects}

In total, twelve subjects (9 males and 3 females; age $56.6 \pm 11.9$ years) were recruited from a Parkinson's disease (PD) dynamic $\left[{ }^{11} \mathrm{C}\right] \mathrm{DPA}-713$ PET research study. Six additional healthy male subjects (age $42.6 \pm 11.2$ years) were also included, out of which five healthy subjects underwent test-retest studies to assess the repeatability of DPA kinetics. The inclusion criteria for the PD cohort are PD clinical diagnosis of 3 to 12 years of duration from onset of symptoms, age 30 to 70 years at time of enrollment, Hoehn and Yahr stages 2-3, and absence of a clinical diagnosis of dementia. Exclusion criteria included subjects receiving dopamine receptor blocking agents or treatment with acetylcholinesterase inhibitors, history of another significant neurological or major psychiatric disorder, or autoimmune disorders within the past 5 years. For screening purpose, all patients including healthy volunteers had a blood sample $(3 \mathrm{~mL})$ collected for TSPO (rs6971) genotype analysis. Three different genotypes are defined: lowaffinity binders (LAB), mixed-affinity binders $(\mathrm{MAB})$, and high-affinity binders (HAB). Patients that are low-affinity binders were excluded from participation. Detailed information on all subjects is shown in Supplementary Table S1.

\section{PET measurements and reconstruction}

For the PET studies, $526.4 \pm 73.6 \mathrm{MBq}(14.2 \pm 1.9 \mathrm{mCi})$ of $\left[{ }^{11} \mathrm{C}\right] \mathrm{DPA}-713$ was administered through bolus-intravenous injection, followed by flushing 10-15 ml of saline solution. PET data were acquired simultaneously after injection in list-mode format on a 4-ring Siemens Biograph $\mathrm{mCT}^{\mathrm{TM}}$ for a total of $90 \mathrm{~min}$. The PET data were reconstructed into 32 dynamic frames $(6 \times 10 \mathrm{~s}, 4 \times 30 \mathrm{~s}, 3 \times 60 \mathrm{~s}, 2 \times 120 \mathrm{~s}$, $5 \times 240 \mathrm{~s}, 12 \times 300 \mathrm{~s})$ using ordered subset expectation maximization (OSEM) with attenuation, scatter, and randoms corrections. Continuous arterial sampling was performed at 15-s intervals for the first $10 \mathrm{~min}$ using an automated fraction collector, followed by five additional samples collected at 20,30, 45, 60, and $90 \mathrm{~min}$ respectively. Each of the blood samples was weighed and counted using a Wizard ${ }^{\circ}$ automatic gamma counter (Perkin Elmer), and then, the activity concentration was calculated. Blood samples drawn at 5, 10, 20, 30, 45, 60, and 90 min post-injection were also used to estimate metabolite fractions using the HPLC method of analysis. The blood time activity curves (TACs) were finally corrected for metabolites, yielding a metabolite-corrected, arterial input function. 
Data analysis and kinetic modeling

Each subject underwent a T1-weighted MRI scan. Inter-frame head motion correction was achieved by rigidly co-registering the individual dynamic PET frames to the last 10 min image set using PMOD (version 3.8; PMOD Technologies Ltd). The resulting dynamic image set was then rigidly registered to the T1-MR image set. Brain regions were delineated on the MRI images using the FreeSurfer software [28], the corresponding volumes of interest (VOIs) were overlaid on the co-registered and motion-corrected dynamic PET images, and finally, the corresponding TACs were deduced.

Kinetic modeling was done for each patient using the Logan $V_{\mathrm{T}}$ model [29]:

$$
\frac{\int_{0}^{t} C(T) d T}{C(t)}=V_{T} \frac{\int_{0}^{t} C_{p}(T) d T}{C(t)}+\text { constant }\left(t \geq t^{*}\right)
$$

where $C(t)$ is the regional time activity curves ( $\left.\mathrm{T}_{t} \mathrm{ACs}\right), C_{p}$ is the input function, and $t^{*}$ is the time at which the plot of $\frac{\int_{0}^{L} C(T) d T}{C(t)}$ versus $\frac{\int_{0}^{t} C_{p}(T) d T}{C(t)}$ reaches linearity. The linearity time was determined using the maximum admissible error criterion as described by Ichise et al. [30]. This automatically searches for the minimum time after which the relative error of every data point in the Logan plot is lower than the given error threshold. A $10 \%$ error criteria was used in this study as also suggested by similar TSPO studies [27]. An example of the Logan fit for a sample patient using both PSAIF and PBIF is shown in Supplementary Figure S7.

Kinetic analysis was performed using the PSAIFs and then repeated using the PBIFs. For each of the selected brain structures (white matter, cerebellum, thalamus, caudate, putamen, pallidum, brainstem, hippocampus, and amygdala), the total volume of distribution $\left(V_{T}\right)$ was estimated with the blood volume fixed to $5 \%$. These brain regions were selected mainly because they show great affinity for $\left[{ }^{11} \mathrm{C}\right] \mathrm{DPA}-713$ binding.

\section{Test-retest repeatability and reliability}

Five healthy volunteers underwent a test-retest within the same day to assess the reproducibility of the kinetic parameters in the brain structures. Kinetic analysis was carried out for all the selected brain regions, for both the test and retest datasets, using the Logan $V_{T}$ model and the corresponding PSAIF's. The repeatability of $V_{T}$ was assessed using the Bland-Altman analysis [31]:

$$
\begin{aligned}
& \% \text { Relative Diff }, D=\frac{\text { Retest }- \text { Test }}{(\text { Retest }+ \text { Test }) / 2} \times 100 \\
& \text { Mean Bias }=\frac{\sum_{n=1}^{N} D}{N}
\end{aligned}
$$

where $N=$ number of subjects

The corresponding 95\% limits of agreement (LOA) and the coefficient of repeatability (CR) between test and retest were determined using:

$$
\begin{aligned}
& L O A=\text { Mean Bias } \pm 1.96 S D \\
& C R=1.96 \times \sqrt{\frac{\sigma^{2}}{N-1}}
\end{aligned}
$$

where $\sigma^{2}$ is the variance of the relative difference, $D$, between the test and retest 
estimates. This represents the value below which the relative difference between test and retest is expected to lie with a $95 \%$ probability $[31,32]$.

\section{Generation of population-based input functions}

The individual PSAIFs were fitted using the "tri-exponential" function and then corrected for metabolites after fitting the later using "Watabe" function (as incorporated in PMOD). The PSAIFs were also fitted with "bi-exponential and gamma" function, but the tri-exponential function gave the best fit for all subjects involved in this study. Sample graphs of the fitted PSAIF, metabolite fraction, and the resulting metabolitecorrected PSAIF are shown in Supplementary Figure S1.

The PBIFs were generated from the metabolite-corrected PSAIFs of all the 18 subjects under review (samples in Supplementary Figure S5A). The metabolite-corrected PSAIFs for all subjects were interpolated to the same time grid (with a step of $1 \mathrm{~s}$ ), and then, their peaks were aligned to the $30 \mathrm{~s}$ time point where the majority of the IF peaks occurred. In order to reduce the influence of subject-induced variation on the generated PBIF, each of the metabolite-corrected PSAIFs was normalized separately by three methods: (a) Weight subject $\times$ Dose $_{\text {Injected, }}$ (b) the corresponding AUC, and (c) Weight $_{\text {subject }} \times$ AUC.

For each subject, PBIF was generated by averaging the normalized PSAIF of the other 17 subjects-leave-one-out procedure [17, 20,33, 34]. Individual subject IFs were then generated by appropriately scaling the PBIF with the corresponding factor, i.e., (a) Weight $_{\text {subject }} \times$ Dose $_{\text {Injected }}$ (b) the corresponding AUC, and (c) Weight ${ }_{\text {subject }} \times$ AUC.

Since the normalized PBIF does not have arterial blood samples, the AUC scaling was done by tail-fitting the normalized PBIF and the PSAIF using the time points 30, 45, 60, and $90 \mathrm{~min}$. Additionally, three pseudo-time points (37.5, 52.5, and $75 \mathrm{~min}$ ) were created as the average of PSAIF at 30 and $45 \mathrm{~min}, 45$ and $60 \mathrm{~min}$, and 60 and 90 min respectively. This was done in order to find an optimal time point which minimizes the difference between the original PSAIF AUC and the PBIF AUC obtained by scaling with one (or two) late blood sample, following a similar approach for TSPO study [27].

The reproducibility of $V_{T}$ using PBIF was assessed using Bland-Altman analysis, with PSAIF values as gold reference. For each structure, the \% relative difference (Relative Diff), $D$, between the parameters was estimated using:

$$
\text { \%Relative Diff, } D=\frac{P_{\mathrm{PBIF}}-P_{\mathrm{PSAIF}}}{P_{\mathrm{PSAIF}}} \times 100
$$

where $P_{\mathrm{PBIF}}$ and $P_{\mathrm{PSAIF}}$ are the kinetic parameters generated by PBIF and PSAIF respectively.

The bias and the corresponding 95\% upper and lower LOA were estimated using Eqs. (3 and 4).

\section{Statistical analysis}

Data were analyzed using the SPSS (IBM SPSS statistics for windows, version 26.0) and Real statistics (http://www.real-statistics.com/) software. Normality of distribution was tested using the Shapiro-Wilk test. The statistical difference between the three normalization techniques was evaluated using the one-way analysis of variance 
(ANOVA). The pairwise $t$-test was also performed as a follow-up test to ANOVA in order to reveal which specific pair of the normalization techniques is significantly different, and Bonferroni correction was applied to correct for the potential error due to multiple testing. In all cases, a $P$-value $<0.05$ was considered to suggest statistical significance.

The major steps involved in this study are represented with a workflow chart in Fig. 1.

\section{Results}

\section{Test-retest repeatability and reliability}

The repeatability of the $V_{\mathrm{T}}$ estimates for all selected brain regions of interest in the test-retest studies are shown in Table 1 and Fig. 2. For all the brain regions of interest, the mean of the $V_{\mathrm{T}}$ estimates from all the healthy volunteers is between 3.18 and 4.91 for test estimates and 3.68 and 5.92 for the retest. The $V_{\mathrm{T}}$ estimates in the retest studies exhibited positive bias (ranging from 20 to 30\%) compared to those deduced from the test studies. A systematic bias is also noticed between the test and the retest results, where all the differences lie above the zero line. The 95\% LOA lies within $\sim 3 \%$ and $\sim 70 \%$ for all regions.

\section{Comparison between PSAIF and PBIF}

Before generating the PBIF from the pool of subjects, we first examined the shape of the PSAIFs between groups (HV versus PD) and genotype (MAB vs HAB). The results are shown in Fig. 2 and Supplementary S2. Visual inspection of the average IF for HV and PD showed no difference between groups (Fig. 3). Also, the log-transformation plot showed no difference in the peak or tail for different groups and genotype (Supplementary Figure S2).

Therefore, the PSAIFs for all the eighteen subjects involved in this study were pooled together to generate the PBIF using the three normalization techniques used in this study. The individual IFs were generated by appropriately scaling the PBIF with the corresponding normalization factor. AUC scaling was done by tail-fitting the

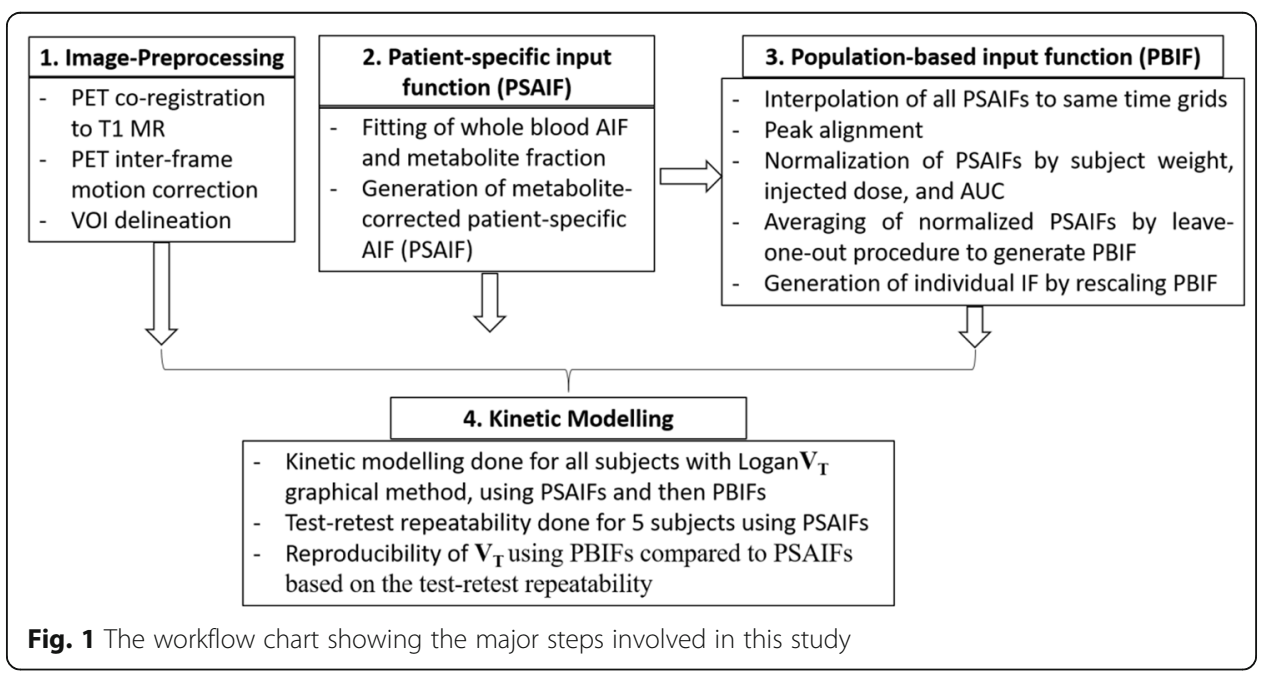


Table 1 Bland-Altman analysis of the variation in $V_{T}$ estimates between the test and retest

\begin{tabular}{llllll}
\hline Regions & Test mean & Retest mean & Mean \% bias \pm SD & 95\% LOA & ICC \\
\hline White matter & $3.74 \pm 2.02$ & $4.43 \pm 2.14$ & $21.43 \pm 15.08$ & -8.74 to 51.60 & 0.93 \\
Cerebellum & $3.61 \pm 1.87$ & $4.50 \pm 2.15$ & $25.69 \pm 14.40$ & -3.12 to 54.50 & 0.88 \\
Thalamus & $4.51 \pm 2.50$ & $5.61 \pm 2.77$ & $26.89 \pm 18.01$ & -9.12 to 62.90 & 0.90 \\
Caudate & $3.16 \pm 1.84$ & $3.68 \pm 2.02$ & $18.52 \pm 12.94$ & -7.36 to 44.40 & 0.95 \\
Putamen & $3.83 \pm 2.12$ & $4.71 \pm 2.31$ & $25.71 \pm 17.47$ & -9.24 to 60.65 & 0.91 \\
Pallidum & $4.06 \pm 2.24$ & $4.83 \pm 2.35$ & $22.70 \pm 20.56$ & -18.41 to 63.82 & 0.91 \\
Brainstem & $4.91 \pm 2.92$ & $5.92 \pm 3.15$ & $23.61 \pm 15.80$ & -7.99 to 55.21 & 0.94 \\
Hippocampus & $4.09 \pm 2.29$ & $5.04 \pm 2.39$ & $26.44 \pm 17.65$ & -8.85 to 61.73 & 0.91 \\
Amygdala & $4.04 \pm 2.35$ & $4.99 \pm 2.42$ & $27.98 \pm 19.40$ & -10.81 to 66.78 & 0.92 \\
\hline
\end{tabular}

normalized PBIF and the PSAIF using the time points 30, 37.5, 45, 52.5, 60, 75, and 90 min. The AUC of the scaled PBIF using the different time points and the original PSAI F were then compared by evaluating the \%error (result in Fig. 4). Although there is no significant difference in the \%error between the different time points, the sample at 75 min yielded the least \%error of $0.53 \%$. Since 75 min was actually an average between the blood collected at 60 and $90 \mathrm{~min}$, the AUC scaling (i.e., scaling with one (or two) late blood sample) in this study was done by tail-fitting the PBIF and the PSAIF using the last $30 \mathrm{~min}$ time points (i.e., between 60 and $90 \mathrm{~min}$ ).

\section{Evaluation of the PBIF and the normalization criteria}

Figure 5 shows the \% difference and the LOAs between the $V_{\mathrm{T}}$ estimates generated by the PSAIF and PBIF for selected brain regions. The comparison is made using PSAIF and the PBIF generated by the three normalization techniques. The mean $\%$ difference is $-10 \%$ for Weight $_{\text {subject }} \times$ Dose $_{\text {Injected }},+8 \%$ for AUC, and $+2 \%$ for Weight $_{\text {subject }} \times$ AUC, while the LOAs lie within $\pm 45 \%$ for Weight $_{\text {subject }} \times$ Dose $_{\text {Injected }} \pm 50 \%$ for AUC, and \pm

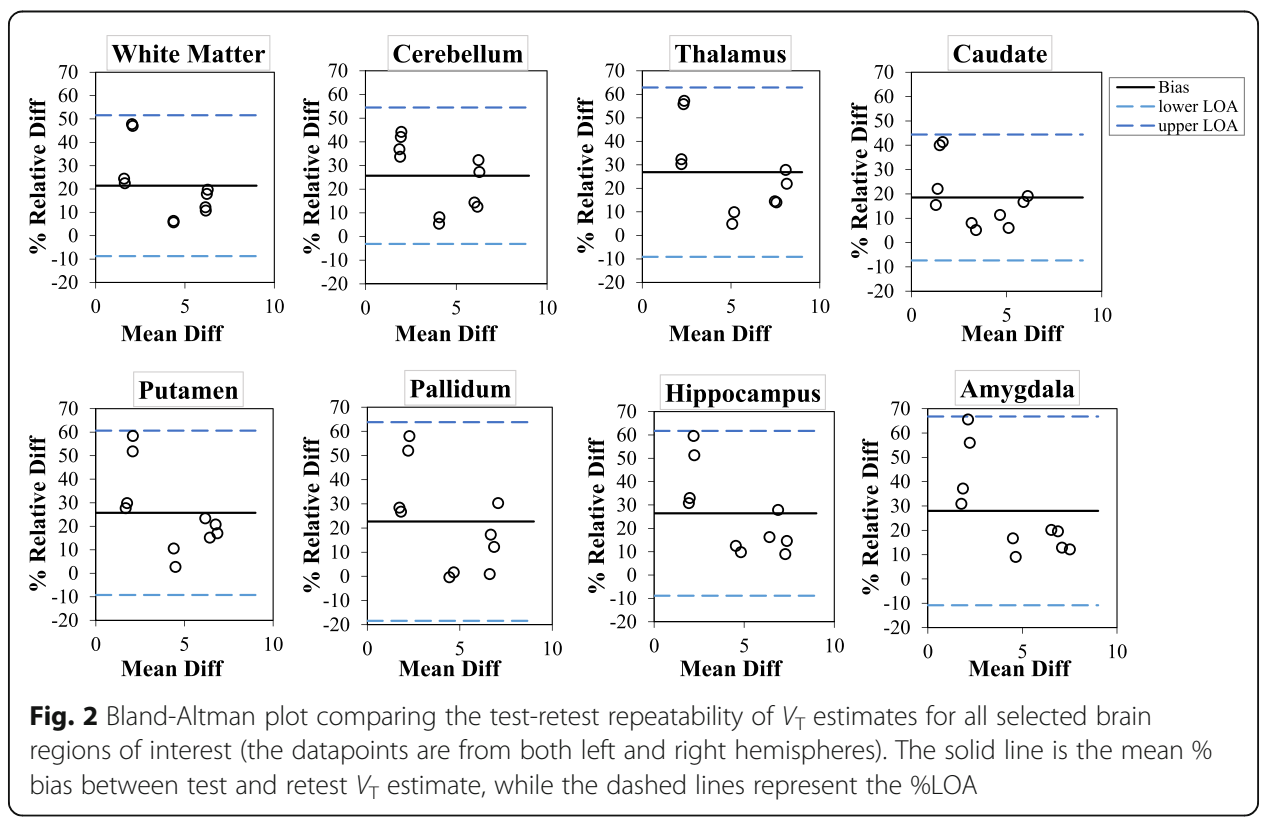




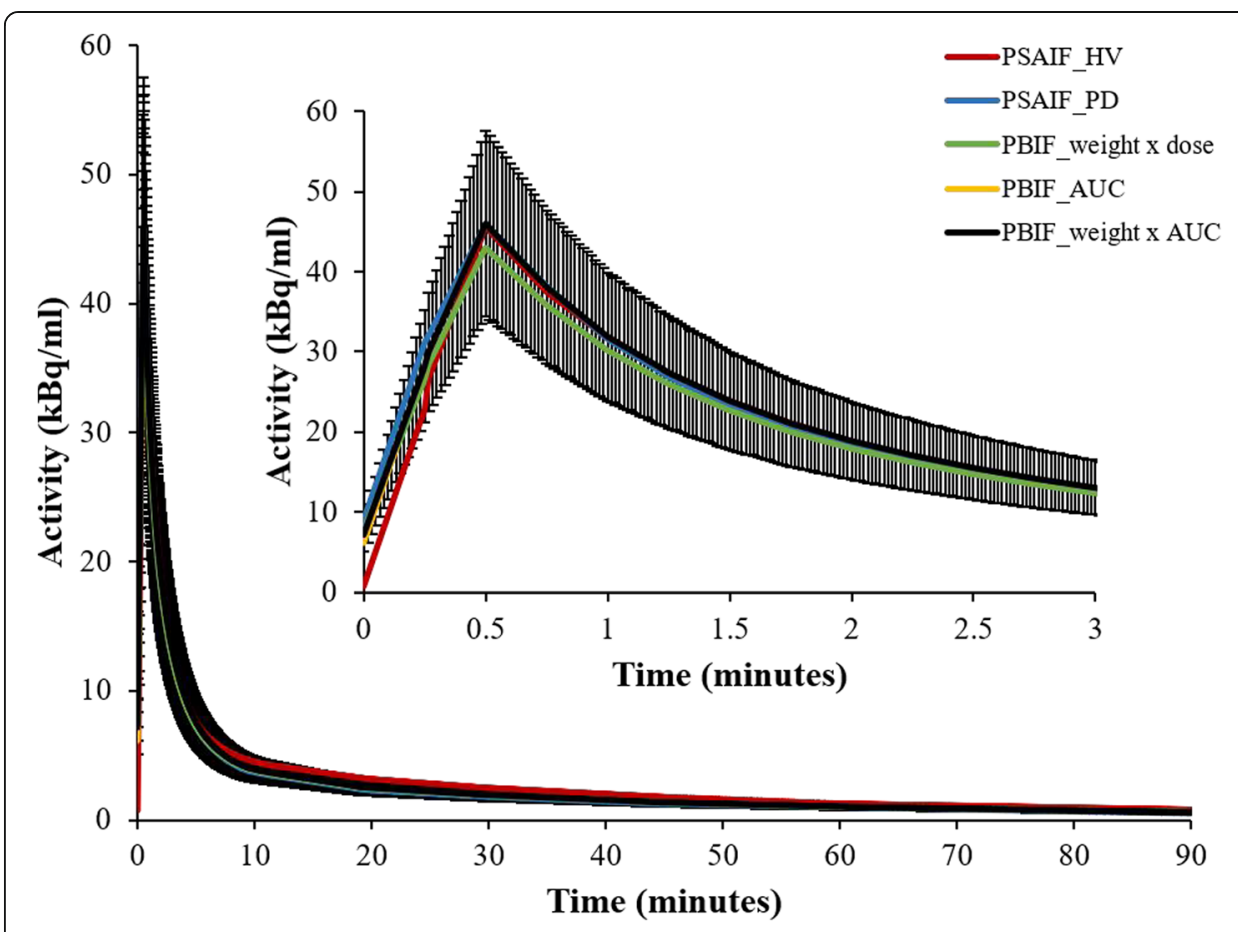

Fig. 3 Comparison between the averaged PSAIFs of HV and PD subjects, and the resulting PBIF generated from the three normalization techniques. The inner plot shows the zoomed IF over the first $3 \mathrm{~min}$. The standard deviation (SD) is shown for the PBIF generated by normalization with Weight subject $_{\text {XAUC. }}$

$38 \%$ for Weight subject $\times A U C$. The ANOVA analysis shows a significant difference between the results generated by the three normalization techniques for all brain structures except the brainstem $(P$-value $=0.095)$. Although for the same brainstem, the pairwise test shows a significant difference between Weight $_{\text {subject }} \times$ Dose $_{\text {Injected }}$ versus AUC $(P$-value $=0.034)$. In all cases, normalizing by Weight subject $\times A U C$ yielded the smallest $\%$ bias and variability (\% bias $= \pm 2 \%$; LOA $= \pm 38 \%$ for all brain regions).

The mean bias $( \pm \mathrm{SD})$ between the PSAIF and PBIF for the $V_{\mathrm{T}}$ generated by normalization with Weight subject $\times A U C$ are shown in Fig. 6 and Table 2 (for all brain regions).

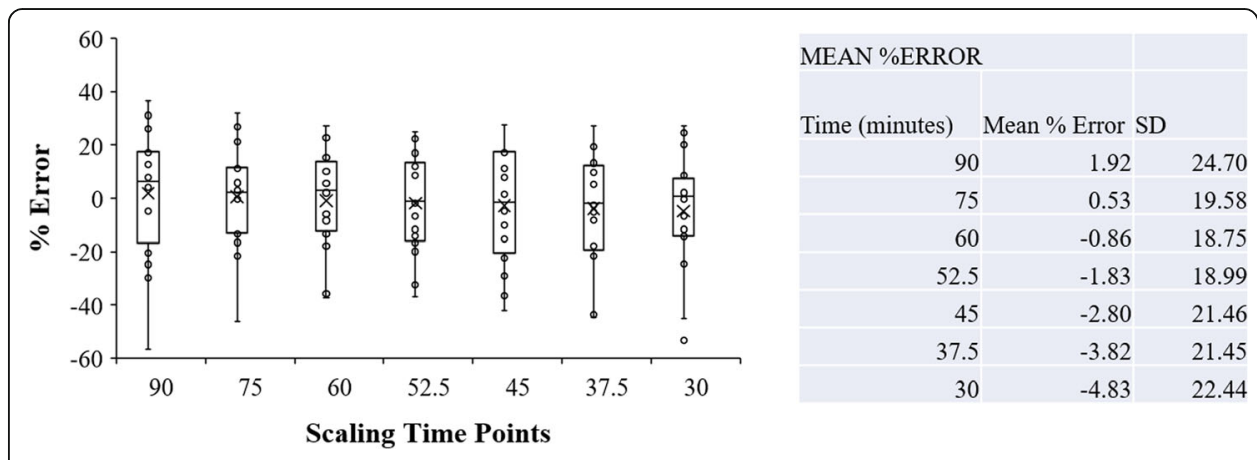

Fig. 4 AUC comparison of the scaled PBIF using the different time points and the original PSAIF. Note that no blood was collected at time points 37.5, 52.5, and 75 min; they were just average time points of 30 and $45 \mathrm{~min}, 45$ and $60 \mathrm{~min}$, and 60 and 90 min respectively 


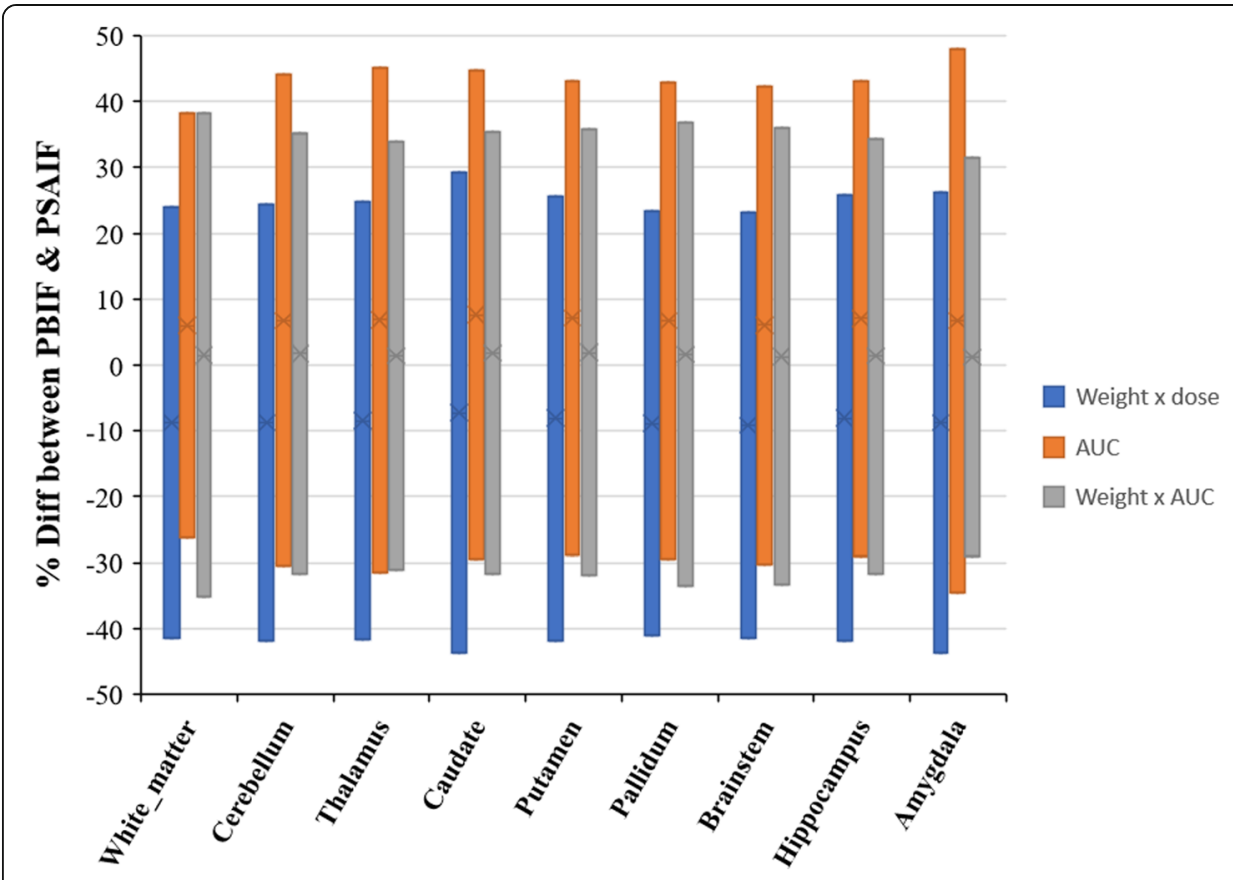

Fig. 5 The \% relative difference in $V_{T}$ and the LOAs between PSAIF and PBIF of some specific structures as generated by the three normalization techniques

The mean bias for $V_{\mathrm{T}}$ lies within $\pm 2 \%$, with amygdala showing the smallest $(1.21 \%)$ deviation and putamen showing the highest (1.91\%). Overall, the 95\% LOA for all brain regions lies within $\pm 38 \%$.

We also evaluated the reproducibility of the $V_{\mathrm{T}}$ generated with PBIF to that of PSAIF using the estimated $t^{*}$, and other goodness of fit criteria (AIC, $R^{2}$, and $\%$ standard error (SE)). The result is shown in Supplementary Figure S8. There is no difference between the $V_{\mathrm{T}} \mathrm{s}$ generated by PBIF and PSAIF based on these criteria.

\section{Agreement of $V_{\mathrm{T}}$ between PSAIF and PBIF (based on disease groups and genotype)}

Finally, we estimated how well the PBIF-kinetics replicates the PSAIF-kinetics based on disease groups (HV versus $\mathrm{PD}$ ) and genotype (MAB versus HAB). Figure 7 shows the Logan $V_{\mathrm{T}}$ values for all subjects calculated with PSAIF and PBIF for the two genotype groups ( $\mathrm{HAB}$ and $\mathrm{MAB}$ ). For all brain regions, the average $V_{\mathrm{T}}$ values obtained from PBIF is slightly higher than PSAIF, but the difference is not significant for each genotype group (\%difference $=4.53 \%, P$-value $=0.73$ for $\mathrm{HAB}$; and $\%$ difference $=0.73 \%, P$-value $=0.96$ for $\mathrm{MAB}$ ). Comparing $\mathrm{MAB}$ to $\mathrm{HAB}$, there is a significant reduction in $V_{\mathrm{T}}$ both with PSAIF and PBIF. PSAIF showed an average reduction of $40 \%$ in $V_{\mathrm{T}}$ across the brain regions, while the average reduction with PBIF is $42 \%$. T-test showed a $P$-value $<0.01$ for all the brain regions, both for PSAIF and PBIF, and on average, the $P$-value for PBIF is about 50\% higher than PSAIF.

Figure 8 shows the difference in Logan $V_{\mathrm{T}}$ values calculated with PSAIF and PBIF between $\mathrm{HV}$ and PD subjects, and also $\mathrm{HAB}$ and MAB groups. The average $V_{\mathrm{T}}$ values are insignificantly higher in PD patients compared to HV. PBIF tend to overestimate the 


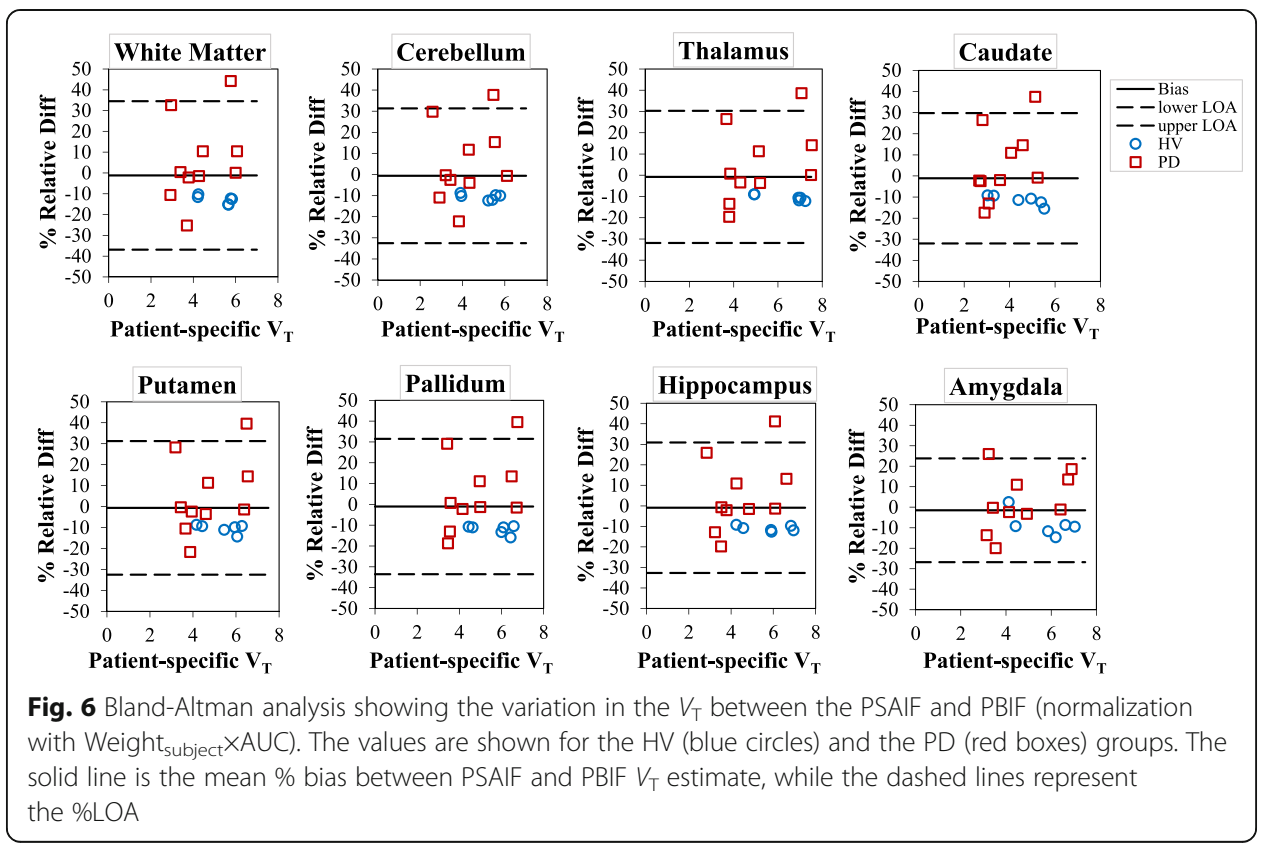

difference between PD and HV for HAB (\%difference $=32.33 \%, P$-value $=0.32$ with PBIF; \%difference $=13.28 \%, P$-value $=0.64$ with PSAIF). However, this difference is underestimated in MAB (\%difference $=6.84 \%, P$-value $=0.77$ with PBIF; \%difference $=$ $20.92 \%, P$-value $=0.55$ with PSAIF) .

\section{Discussion}

Several studies have shown the feasibility to image neuroinflammation in multiple sclerosis (MS), Parkinson's disease (PD), and Alzheimer's disease (AD) using $\left[{ }^{11} \mathrm{C}\right] \mathrm{DPA}$ 713 PET for quantifying differences between patients and controls [4, 9-12]. Accurate quantification of tracer uptake and metabolism in the tissue through kinetic modeling often requires blood sampling [13] or some alternative approach such as simplified reference modeling [35, 36], cluster analysis [35, 37], or image-derived input function (IDIF) techniques. The apparent limitations of these approaches $[15-17,19,21,38]$ are giving way to the exploration of the population-based input function (PBIF) approach as a more quantitatively reliable and less invasive alternative.

Table 2 Bland-Altman analysis of the variation in $V_{T}$ estimate between the PSAIF and PBIF (normalization with Weight $_{\text {subject }} \times A \cup C$ )

\begin{tabular}{lll}
\hline Regions & Mean \% bias \pm SD & 95\% LOA \\
\hline White matter & $1.51 \pm 18.74$ & -35.22 to 38.23 \\
Cerebellum & $1.73 \pm 17.09$ & -31.75 to 35.22 \\
Thalamus & $1.45 \pm 16.57$ & -31.02 to 33.93 \\
Caudate & $1.80 \pm 17.14$ & -31.79 to 35.40 \\
Putamen & $1.91 \pm 17.27$ & -31.96 to 35.77 \\
Pallidum & $1.57 \pm 17.95$ & -33.60 to 36.75 \\
Brainstem & $1.28 \pm 17.67$ & -33.35 to 35.92 \\
Hippocampus & $1.36 \pm 16.86$ & -31.67 to 34.41 \\
Amygdala & $1.21 \pm 15.42$ & -29.01 to 31.45 \\
\hline
\end{tabular}




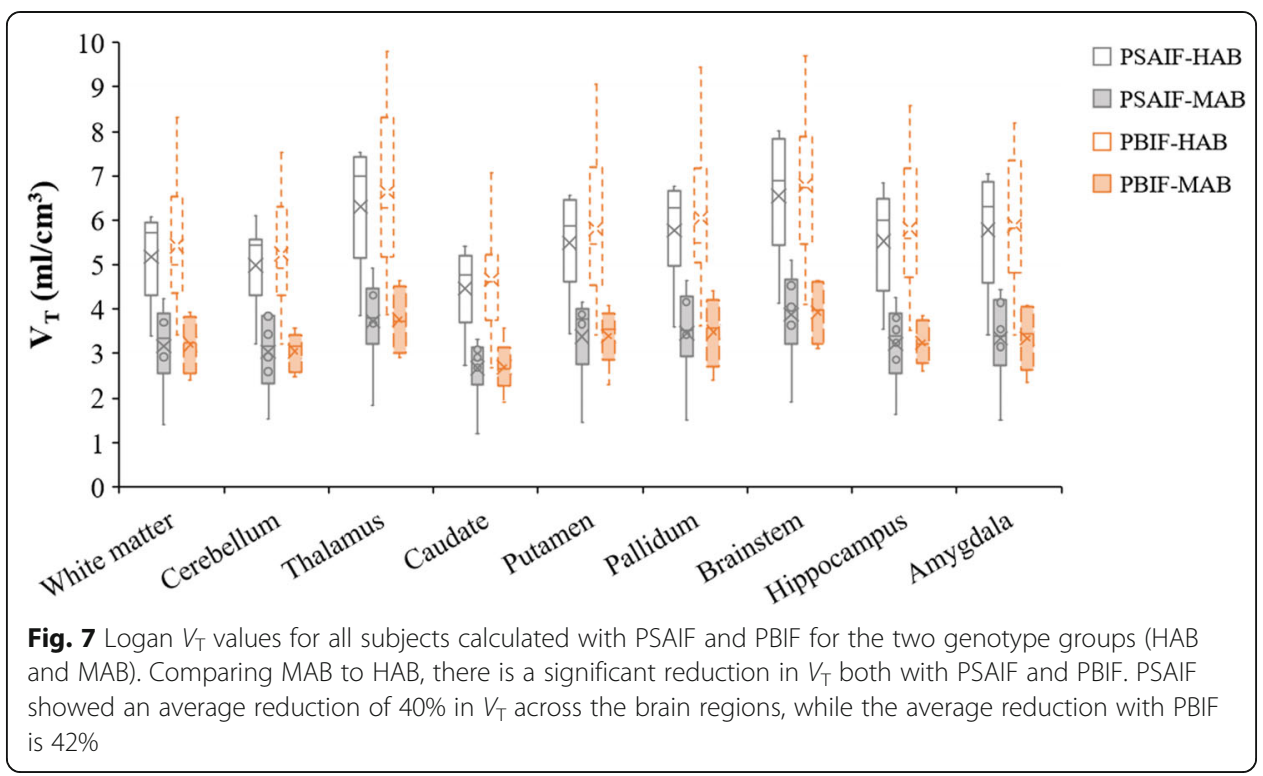

In this study, we have assessed the reproducibility of kinetic analysis of $\left[{ }^{11} \mathrm{C}\right] \mathrm{DPA}-713$ dynamic PET images using PBIF, compared to PSAIF, in a cohort of subjects with Parkinson's disease and healthy volunteers. The major steps involved in this study are represented in a workflow chart in Fig. 1. The repeatability of the $V_{\mathrm{T}}$ estimate was also assessed in a cohort of healthy volunteers that underwent a test-retest $\left[{ }^{11} \mathrm{C}\right] \mathrm{DPA}-713$ dynamic PET within the same day. Kinetic analysis with PSAIF was determined to be reproducible with PBIF if the corresponding LOA are within those of the test-retest study.

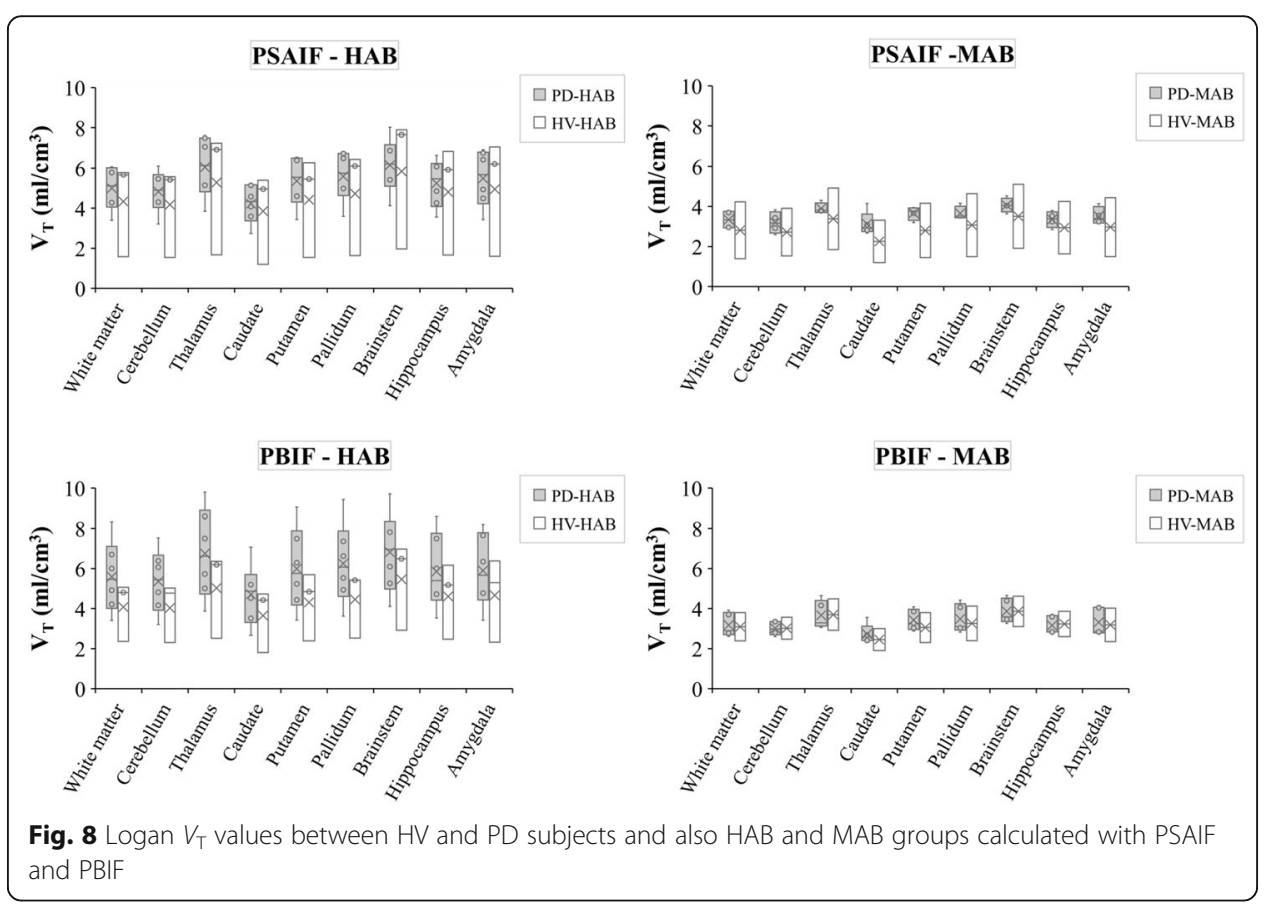




\section{Test-retest repeatability}

The test-retest repeatability study of the $\left[{ }^{11} \mathrm{C}\right] \mathrm{DPA}-713$ uptake exhibited systematic increase in uptake values between test and retest (Fig. 2 and supplementary Figure S3) although the cause of this systematic bias is yet to be fully explored in same day testretest repeatability studies because most repeatability studies are done days or even weeks apart $[39,40]$. Few recent studies that performed same day test-retest have reported the same systematic bias, and they suggested that the possible explanation to this systematic bias could be due to hormone-mediated changes in TSPO expression, tonic changes due to scan-related stress/anxiety, or alteration in blood cholesterol due to food intake between the test and retest scans [39, 41-43]. While performing test and retest studies under similar conditions on different days could eliminate this bias [40], other parameters such as alteration in TSPO density due to chronic disease as well as non-disease-related factors may be difficult to control [43].

One potential approach to compensate for the systematic bias between the test and retest studies is by normalization by the corresponding kinetic parameters of the GM as suggested by past studies [39, 43-45] and also shown by this study (Supplementary Figure S3 and S4). Without GM normalization, the \% relative difference between test and retest $V_{\mathrm{T}}$ values lies significantly above the zero line for all structures, indicating that retest values are always higher than test values. But with GM normalization, the \% relative difference is symmetric about the zero line. The mean \% Diff, the LOA and the $\mathrm{CR}$ are also significantly reduced, thereby improving repeatability. However, past studies involving gray matter normalization was validated in a clinical population (HIV with associated cognitive deficits) with regional inflammation. We believe there is no sufficient justification of using the GM normalization approach for PD cohorts since neuroinflammation can occur in any brain region, and therefore, we elected not to adopt it.

Another possible explanation for the high test-retest bias as reported in this study is the small regions of interest used and the relatively small number of healthy subjects $(n=5)$ who underwent the test-retest scans. This is in agreement with a TSPO study from Jucaite et al. [39] which showed that the mean bias between test and retest was low in large brain regions (such as the whole brain, cortical gray matter and white matter) but high in smaller brain regions. They also attributed the large variability to the limited sample size, and this was also confirmed by Plaven-Sigray et al. [46] who estimated a test-retest variability in six healthy controls and obtained a variability of about $21 \%$ in $V_{\mathrm{T}}$. In fact, Collste et al. [40] carried out a test-retest study with six subjects examined on the same day and other six subjects examined $2-5$ days apart. The \% bias was within $14-27 \%$ for subjects examined within the same day and $0.2-8.4 \%$ for subjects examined 2-5 days apart. We therefore recommend that more research still needs to be done in order to fully understand the effect of small sample size, small brain regions of interest, as well as days between test and retest scans on the test-retest reproducibility.

\section{Generation and evaluation of the PBIF}

The PBIF was generated from the PSAIF of all 18 subjects after examining the shape of the PSAIFs between groups (HV versus PD) and genotype (MAB vs HAB). This was motivated by Owen et al. [47, 48], who demonstrated that the second generation TSPO 
tracers target two binding sites in humans, which leads to three affinity patterns: low-, high-, and mixed-affinity binders (LABs, HABs, and MABs respectively). Past researches have shown that this variability in binding affinity has a major influence on the kinetic parameters where the values for HABs could be approximately twice that of MABs [48, 49]. For $\left[{ }^{11} \mathrm{C}\right]$-DPA-713 dynamic PET studies, Coughlin et al. [43] argued that those genotypes as well as other unknown physiological factors have varying degrees of influence on the global TSPO changes in the brain, thereby hindering accurate PET analysis, even among individuals with the same genotype. This was also confirmed in other TSPO studies [39, 45, 50]. Our results (Fig. 3 and Supplementary Figure S2) however showed no significant difference in PSAIF between the groups, and so, all subject PSAIFs were included in the generation of the PBIF. This was also in agreement with other studies [27, 51].

Ye et al. [52] opined that the bias in kinetic parameter estimation in direct reconstruction with PBIF depends on the normalization and scaling technique used. In this study, we have assessed and compared three normalization approaches: (a) Weight sub- $_{\text {- }}$ $j_{\text {ject }} \times$ Dose $_{\text {Injected }}$ (b) AUC, and (c) Weight subject $\times A U C$. These normalization techniques were chosen because they reduced the influence of subject-induced variation on the generated PBIF. We also normalized the PSAIFs by different methods (as shown in supplementary Figure S6 and Table S2). However, since our aim is to reduce patient variability in the generation of the PBIF, we decided to go with the above stated normalization techniques. An example of the normalized PSIFs and the resulting PBIF are shown in Supplementary Figure S5. The performance of these techniques was evaluated using the percent relative difference between the PSAIF- and PBIF-derived $V_{\mathrm{T}}$ in selected brain regions (Fig. 5). There is a significant difference between the three normalization techniques for all brain structures except the brainstem.

Several normalization techniques have been reported in the literature which include traditional scaling using blood samples by correlating the measured plasma activity at a given time-point with the AUC [17]; correlation of the PBIF with AIF at any time-point using venous samples [19]; by accounting for injected dose and weight [20, 21]; non-invasive scaling using individual parameters like weight, body surface area (BSA), and lean body mass (LBM) [17]. In this study, we have assessed the three aforementioned normalization approaches. Subsequently, a subject IF was deduced by scaling the PBIF by his/her weight and injected dose. In the case of AUC normalization, this was measured after scaling the PBIF by the ratio of the average activity concentration of blood samples acquired over the last $30 \mathrm{~min}$ of the dynamic scan (i.e., between 60 and $90 \mathrm{~min}$ ) and that of the tail of the PBIF over the same time frames. This setting was used as this best minimizes the error between PSAIF AUC and the scaled PBIF (Fig. 4).

Precisely, the AUC between PSAIF and PBIF was minimized by scaling the PBIF with an arterial blood value at $75 \mathrm{~min}$, as also recommended by past similar TSPO studies $[27,51]$. It has been shown that venous blood samples may practically be used instead for scaling purpose since arterial and venous blood tend to reach equilibrium at about 30-45 min post-injection time [17]. Although this was not tested in this work, but similar TSPO studies have also found that PBIF can be appropriately scaled using one blood sample $[27,51]$. Since the utmost aim is to potentially alleviate the need for arterial blood sampling, the AUC component of the 
PBIF normalization can be obtained by scaling the normalized PBIF by the ratio of the average activity concentration of blood samples (possibly venous blood) acquired over the last $30 \mathrm{~min}$ of the dynamic scan (i.e., between 60 and $90 \mathrm{~min}$ ) and that of the tail of the PBIF over the same time frames (as was done in this study). However, more relevant clinical studies need to be conducted to establish a correlation between activity concentration in arterial and venous blood samples at these latter time points of the scan.

In this study, normalization by Weight subject $\times$ AUC yielded the smallest $\%$ bias $( \pm 2 \%)$ and variability (LOAs $\pm 38 \%$ ) between PBIF and PSAIF (Fig. 5 ). $V_{\mathrm{T}}$ measured with PBIF showed good reproducibility (LOA of $\pm 38 \%$ ) but with a positive bias ( $\pm 2 \%$ ) (Fig. 6 and Table 2). These were also in agreement with the findings of Lavisse et al. [19]. As a final note, the reproducibility of the PBIF-based $V_{\mathrm{T}}$ estimates compared with PSAIFbased $V_{\mathrm{T}}$ fall well within the test-retest results (Table 1), hence showing the feasibility of $\left[{ }^{11} \mathrm{C}\right]$-DPA-713 PET kinetic modeling using PBIF.

PBIF was able to reproduce the PSAIF kinetic results because of the similar patterns in average AIF between disease groups and genotypes (Fig. 3 and Supplementary Figure S2). Although we expect that PBIF cannot exactly reproduce the peak and shape of the PSAIF (as shown in Supplementary Figure S2), but having a similar AUC between PSAIF and PBIF will result in less bias in kinetic parameter estimation. That is why previous studies have recommended that Logan $V_{\mathrm{T}}$ method is more suitable for PBIF than 2-tissue compartment model because Logan $V_{\mathrm{T}}$ relies on the AUC of the IF and therefore less sensitive to the shape $[20,53]$.

A major limitation for this study is the relatively small sample size $(n=18)$, even though our findings are in agreement with previous results of smaller $(n=9)$ [19] and larger $(n=42)$ [20] sample sizes. A common factor among these studies is the normalization of the individual input functions to remove variabilities in the PBIF. This might suggest that the efficiency of the PBIF in accurately estimating the kinetic parameters depends less on the sample size used but more on the normalization. This was also consolidated by Ye et al. [52] who opined that the bias in kinetic parameter estimation in direct reconstruction with PBIF was mostly due to inaccuracy in normalization and scaling.

\section{Conclusion}

This study demonstrated the feasibility of $\left[{ }^{11} \mathrm{C}\right]$-DPA-713 PET kinetic modeling using PBIF with Logan graphical analysis, thus potentially alleviating the need for arterial blood sampling. Moreover, it was shown that the optimal result in terms of kinetic parameter accuracy was obtained when the PSAIFs were normalized with Weight $_{\text {subject }} \times$ AUC.

\section{Supplementary Information}

The online version contains supplementary material available at https://doi.org/10.1186/s40658-021-00381-8.

Additional file 1: Table S1. Demographic information of all the PD patients and healthy volunteers (HV) included in this study. Figure S1. Sample graphs of the fitted PSAIF, metabolite fraction and the resulting metabolite corrected PSAIF. Figure S2. Comparison between the PSAIF of healthy volunteers (HV) and Parkinson's disease

(PD) patients based on genotype. Log transformation was applied on the plot A, and it shows the average PSAIF 
for each group. Plots B and C show the box plots for the individual subjects in each group. The results demonstrate no substantial differences in the peak and the tail of the IFs across the groups. Therefore, all IFs were used to estimate the PBIF (shown in black). Figure S3. Bland-Altman plot comparing the test-retest repeatability of Vt estimates for all selected brain regions of interest: (A) without GM normalization and (B) with GM normalization respectively. The solid line is the mean \% bias between test and retest $\mathrm{V}_{T}$ estimate, while the doted and dashed lines represent the \%Cl and \%LOA respectively. Figure S4. Overlaid normalized PSAIFs from all 18 patients (A) and the resulting PBIF generated by normalization with Weight subject $\times A U C$ (B). The zoomed PBIF over the first 5 minutes is also shown. In (b), the blue points are the mean PBIF while the red points are the standard error of the mean (SEM). Figure S5. Example of the Logan VT plot generated by the patient-specific input function (upper row) and the population-based input function (lower row). Figure S6. Comparing the PBIF-estimated $V_{T}$ and PSAIF-estimated VT using the goodness of fit criteria.

\section{Acknowledgements}

We want to sincerely appreciate Cynthia Fox in the Department of Radiology, Weill Cornell Medical College, New York, USA, for editing this manuscript.

\section{Authors' contributions}

MIA and SAN conceived the idea, designed the study, and participated in data acquisition. MIA, SAZ, SP, NAK, and SAN carried out the data processing and analysis. JB and AN performed the radiotracer production and metabolite analysis. PDM was involved in the arterial line and procedure. AG participated in the study design and collaboration. SAG, AR, and $\mathrm{CH}$ are the principal investigators for the DPA studies; they are in charge of funding acquisition and subject accrual. MIA wrote the manuscript. All authors reviewed the manuscript and approved the final version for submission.

\section{Funding}

This study was funded by the National Center for Advancing Translational Sciences of the National Institutes of Health under Award Numbers UL1TR000457 and RO1 NS104283.

\section{Declarations}

\section{Competing interest}

The authors declare that they have no competing interest.

\section{Ethics approval and consent to participate}

All procedures performed in the human studies contained in this work were in accordance with the ethical standards of the institutional review board at the Weill Cornell Medical College, New York, USA.

\section{Consent for publication}

Informed consent was obtained from all individual participants included in this study.

\section{Author details}

'Department of Radiology, Weill Cornell Medical College, New York, NY 10021, USA. ²Department of Neurology, Weill Cornell Medical College, New York, NY 10021, USA. ${ }^{3}$ Feil Family Brain and Mind Institute, Weill Cornell Medical College, New York, NY 10021, USA.

Received: 20 January 2021 Accepted: 29 March 2021

Published online: 29 April 2021

\section{References}

1. Cumming P, Burgher B, Patkar O, Breakspear M, Vasdev N, Thomas P, et al. Sifting through the surfeit of neuroinflammation tracers. J Cereb Blood Flow Metab. 2018;38(2):204-24. https://doi.org/10.1177/0271678X17748786.

2. Hagens M, Berckel B, Barkhof F. Novel MRI and PET markers of neuroinflammation in multiple sclerosis. Curr Opin Neurol. 2016;29(3):229-36. https://doi.org/10.1097/WCO.0000000000000326.

3. Airas L, Rissanen E, Rinne J. Imaging of microglial activation in MS using PET: research use and potential future clinical application. Mult Scler. 2017;23(4):496-504. https://doi.org/10.1177/1352458516674568.

4. Boutin H, Chauveau F, Thominiaux C, Gregoire MC, James ML, Trebossen R, et al. C-11-DPA-713: a novel peripheral benzodiazepine receptor PET ligand for in vivo imaging of neuroinflammation. J Nucl Med. 2007;48(4):573-81. https:// doi.org/10.2967/jnumed. 106.036764.

5. Fookes CJR, Pham TQ, Mattner F, Greguric I, Loc'h C, Liu X, et al. Synthesis and biological evaluation of substituted F-18 imidazo 1,2-a pyridines and F-18 pyrazolo 1,5-a pyrimidines for the study of the peripheral benzodiazepine receptor using positron emission tomography. J Med Chem. 2008;51(13):3700-12. https://doi.org/10.1021/jm7014556.

6. Kreisl WC, Fujita M, Fujimura Y, Kimura N, Jenko KJ, Kannan P, et al. Comparison of [(11)C]-(R)-PK 11195 and [(11)C]PBR28, two radioligands for translocator protein $(18 \mathrm{kDa})$ in human and monkey: implications for positron emission tomographic imaging of this inflammation biomarker. Neuroimage. 2010;49(4):2924-32. https://doi.org/10.101 6/j.neuroimage.2009.11.056.

7. James ML, Fulton RR, Vercoullie J, Henderson DJ, Garreau L, Chalon S, et al. DPA-714, a new translocator protein-specific ligand: synthesis, radiofluorination, and pharmacologic characterization. J Nucl Med. 2008;49(5):814-22. https://doi.org/1 0.2967/jnumed.107.046151.

8. Endres CJ, Pomper MG, James M, Uzuner O, Hammoud DA, Watkins CC, et al. Initial evaluation of C-11-DPA-713, a novel TSPO PET ligand, in humans. J Nucl Med. 2009;50(8):1276-82. https://doi.org/10.2967/jnumed.109.062265. 
9. Doorduin J, Klein HC, Dierckx RA, James M, Kassiou M, de Vries EFJ. C-11 -DPA-713 and F-18 -DPA-714 as new PET tracers for TSPO: a comparison with C-11 -(R)-PK11195 in a rat model of herpes encephalitis. Mol Imaging Biol. 2009; 11(6):386-98. https://doi.org/10.1007/s11307-009-0211-6.

10. Rosenberg P, Endres C, Lyketsos C, Coughlin J, Kassiou M, Pomper M. Quantifying translocator protein (TSPO) in Alzheimer's disease and cognitively healthy older persons with 11C-DPA-713 PET imaging. Alzheimers Dement. 2011;7: S725. https://doi.org/10.1016/.jalz.2011.05.2091.

11. Zimmer ER, Leuzy A, Benedet AL, Breitner J, Gauthier S, Rosa-Neto P. Tracking neuroinflammation in Alzheimer's disease: the role of positron emission tomography imaging. J Neuroinflammation. 2014;11(1):120. https://doi.org/10.1186/1742-2 094-11-120.

12. Terada T, Yokokura M, Yoshikawa E, Futatsubashi M, Kono S, Konishi T, et al. Extrastriatal spreading of microglial activation in Parkinson's disease: a positron emission tomography study. Ann Nucl Med. 2016;30(8):579-87. https://doi. org/10.1007/s12149-016-1099-2.

13. Bentourika M. Kinetic modeling of PET-FDG in the brain without blood sampling. Comput Med Imaging Graph. 2006; 30(8):447-51. https://doi.org/10.1016/j.compmedimag.2006.07.002.

14. Kang Y, Mozley PD, Verma A, Schlyer D, Henchcliffe C, Gauthier SA, et al. Noninvasive PK11195-PET Image analysis techniques can detect abnormal cerebral, microglial activation in Parkinson's disease. J Neuroimaging. 2018;28(5):496505. https://doi.org/10.1111/jon.12519.

15. Watabe H, Channing MA, Riddell C, Jousse F, Libutti SK, Carrasquillo JA, et al. Noninvasive estimation of the aorta input function for measurement of tumor blood flow with. IEEE Trans Med Imaging. 2001;20(3):164-74. https://doi.org/10.11 09/42.918468.

16. Mourik JEM, van Velden FHP, Lubberink M, Kloet RW, van Berckel BNM, Lammertsma AA, et al. Image derived input functions for dynamic High Resolution Research Tomograph PET brain studies. Neuroimage. 2008;43(4):676-86. https:// doi.org/10.1016/j.neuroimage.2008.07.035.

17. Zanotti-Fregonara P, Hines CS, Zoghbi SS, Liow JS, Zhang Y, Pike WW, et al. Population-based input function and imagederived input function for C-11 (R)-rolipram PET imaging: methodology, validation and application to the study of major depressive disorder. Neuroimage. 2012;63(3):1532-41. https://doi.org/10.1016/..neuroimage.2012.08.007.

18. Brock CS, Young H, Osman S, Luthra SK, Jones T, Price PM. Glucose metabolism in brain tumours can be estimated using [18F] 2-fluorodeoxyglucose positron emission tomography and a population-derived input function scaled using a single arterialised venous blood sample. Int J Oncol. 2005;26(5):1377-83. https://doi.org/10.3892/ijo.26.5.1377.

19. Lavisse S, Garcia-Lorenzo D, Peyronneau MA, Bodini B, Thiriez C, Kuhnast B, et al. Optimized quantification of translocator protein radioligand F-18-DPA-714 uptake in the brain of genotyped healthy volunteers. J Nucl Med. 2015; 56(7):1048-54. https://doi.org/10.2967/jnumed.115.156083.

20. Zanotti-Fregonara P, Hirvonen J, Lyoo CH, Zoghbi SS, Rallis-Frutos D, Huestis MA, et al. Population-based input function modeling for F-18 FMPEP-d(2), an inverse agonist radioligand for cannabinoid CB1 receptors: validation in clinical studies. PLoS One. 2013;8(4):e60231. https://doi.org/10.1371/journal.pone.0060231.

21. MacAskill MG, Walton T, Williams L, Morgan TEF, Alcaide-Corral CJ, Dweck MR, et al. Kinetic modelling and quantification bias in small animal PET studies with [18F]AB5186, a novel 18 kDa translocator protein radiotracer. PLoS One. 2019;14(5): e0217515. https://doi.org/10.1371/journal.pone.0217515.

22. Wakita K, Imahori Y, Ido T, Fujii R, Horii H, Shimizu M, et al. Simplification for measuring input function of FDG PET: investigation of 1-point blood sampling method. J Nucl Med. 2000;41(9):1484-90.

23. Vriens D, de Geus-Oei L-F, Oyen WJG, Visser EP. A curve-fitting approach to estimate the arterial plasma input function for the assessment of glucose metabolic rate and response to treatment. J Nucl Med. 2009;50(12):1933-9. https://doi. org/10.2967/jnumed.109.065243.

24. Tsuchida T, Sadato N, Yonekura Y, Nakamura S, Takahashi N, Sugimoto K, et al. Noninvasive measurement of cerebral metabolic rate of glucose using standardized input function. J Nucl Med. 1999;40(9):1441-5.

25. Takikawa S, Dhawan V, Spetsieris P, Robeson W, Chaly T, Dahl R, et al. Noninvasive quantitative fluorodeoxyglucose PET studies with an estimated input function derived from a population-based arterial blood curve. Radiology. 1993;188(1): 131-6. https://doi.org/10.1148/radiology.188.1.8511286.

26. Takikawa S, Dhawan V, Chaly T, Robeson W, Dahl R, Zanzi I, et al. Input functions for 6-[fluorine-18]fluorodopa quantitation in parkinsonism: comparative studies and clinical correlations. J Nucl Med. 1994;35(6):955-63.

27. Mabrouk R, Strafella AP, Knezevic D, Ghadery C, Mizrahi R, Gharehgazlou A, et al. Feasibility study of TSPO quantification with [18F]FEPPA using population-based input function. PLoS One. 2017;12(5):e0177785. https://doi.org/10.1371/journal. pone. 0177785

28. Fischl B. FreeSurfer. Neuroimage. 2012;62(2):774-81. https://doi.org/10.1016/j.neuroimage.2012.01.021.

29. Logan J, Fowler JS, Volkow ND, Wolf AP, Dewey SL, Schlyer DJ, et al. Graphical analysis of reversible radioligand binding from time-activity measurements applied to [N-11C-methyl]-(-)-cocaine PET studies in human subjects. J Cereb Blood Flow Metab. 1990;10(5):740-7. https://doi.org/10.1038/jcbfm.1990.127.

30. Ichise M, Toyama H, Innis RB, Carson RE. Strategies to improve neuroreceptor parameter estimation by linear regression analysis. J Cereb Blood Flow Metab. 2002;22(10):1271-81. https://doi.org/10.1097/01.WCB.0000038000.34930.4E,

31. Bland JM, Altman DG. Statistical methods for assessing agreement between two methods of clinical measurement. Lancet. 1986;1(8476):307-10

32. Schwartz J, Humm JL, Gonen M, Kalaigian H, Schoder H, Larson SM, et al. Repeatability of SUV measurements in serial PET. Med Phys. 2011;38(5):2629-38. https://doi.org/10.1118/1.3578604.

33. Karakatsanis N, Zhou Y, Lodge M, Casey M, Wahl R, Subramanian R, et al. Clinical whole-body PET Patlak imaging 6090min post-injection employing a population-based input function. J Nucl Med. 2015;56:1786.

34. Meyer PT, Circiumaru V, Cardi CA, Thomas DH, Bal H, Acton PD. Simplified quantification of small animal [18F]FDG PET studies using a standard arterial input function. Eur J Nucl Med Mol Imaging. 2006;33(8):948-54. https://doi.org/10.1007/ s00259-006-0121-7.

35. Arlicot N, Vercouillie J, Ribeiro MJ, Tauber C, Venel Y, Baulieu JL, et al. Initial evaluation in healthy humans of F-18 DPA714, a potential PET biomarker for neuroinflammation. Nucl Med Biol. 2012;39(4):570-8. https://doi.org/10.1016/j. nucmedbio.2011.10.012. 
36. Golla SSV, Boellaard R, Oikonen V, Hoffmann A, van Berckel BNM, Windhorst AD, et al. Quantification of F-18 DPA-714 binding in the human brain: initial studies in healthy controls and Alzheimer's disease patients. J Cereb Blood Flow Metab. 2015;35(5):766-72. https://doi.org/10.1038/jcbfm.2014.261.

37. Ribeiro M-J, Vercouillie J, Debiais S, Cottier J-P, Bonnaud I, Camus V, et al. Could 18 F-DPA-714 PET imaging be interesting to use in the early post-stroke period? EJNMMI Res. 2014;4(1):28. https://doi.org/10.1186/s13550-014-0028-4

38. Hoekstra CJ, Hoekstra OS, Lammertsma AA. On the use of image-derived input functions in oncological fluorine-18 fluorodeoxyglucose positron emission tomography studies. Eur J Nucl Med. 1999;26(11):1489-92. https://doi.org/10.1 007/s002590050484.

39. Jučaite A, Cselényi Z, Arvidsson A, Åhlberg G, Julin P, Varnäs K, et al. Kinetic analysis and test-retest variability of the radioligand [11C](R)-PK11195 binding to TSPO in the human brain - a PET study in control subjects. EJNMMI Res. 2012 2(1):15. https://doi.org/10.1186/2191-219X-2-15.

40. Collste K, Forsberg A, Varrone A, Amini N, Aeinehband S, Yakushev I, et al. Test-retest reproducibility of [(11)C]PBR28 binding to TSPO in healthy control subjects. Eur J Nucl Med Mol Imaging. 2016;43(1):173-83. https://doi.org/10.1007/ s00259-015-3149-8.

41. Drugan RC. Peripheral benzodiazepine receptors: molecular pharmacology to possible physiological significance in stress-induced hypertension. Clin Neuropharmacol. 1996;19(6):475-96. https://doi.org/10.1097/00002826-19961906000002.

42. Gavish M, Bachman I, Shoukrun R, Katz Y, Veenman L, Weisinger G, et al. Enigma of the peripheral benzodiazepine receptor. Pharmacol Rev. 1999;51(4):629-50.

43. Coughlin JM, Wang Y, Ma S, Yue C, Kim PK, Adams AV, et al. Regional brain distribution of translocator protein using [11C]DPA-713 PET in individuals infected with HIV. J Neurovirol. 2014;20(3):219-32. https://doi.org/10.1007/s13365-0140239-5.

44. Herranz E, Gianni C, Louapre C, Treaba CA, Govindarajan ST, Ouellette R, et al. Neuroinflammatory component of gray matter pathology in multiple sclerosis. Ann Neurol. 2016;80(5):776-90. https://doi.org/10.1002/ana.24791.

45. Vera JH, Guo Q, Cole JH, Boasso A, Greathead L, Kelleher P, et al. Neuroinflammation in treated HIV-positive individuals. Neurology. 2016;86(15):1425-32. https://doi.org/10.1212/WNL.0000000000002485.

46. Plavén-Sigray P, Matheson GJ, Cselényi Z, Jucaite A, Farde L, Cervenka S. Test-retest reliability and convergent validity of (R)-[11C]PK11195 outcome measures without arterial input function. EJNMMI Res. 2018;8(1):102. https://doi.org/10.1186/ s13550-018-0455-8.

47. Owen DRJ, Gunn RN, Rabiner EA, Bennacef I, Fujita M, Kreisl WC, et al. Mixed-affinity binding in humans with 18-kDa translocator protein ligands. J Nucl Med. 2011;52(1):24-32. https://doi.org/10.2967/jnumed.110.079459.

48. Owen DR, Guo Q, Kalk NJ, Colasanti A, Kalogiannopoulou D, Dimber R, et al. Determination of [(11)C]PBR28 binding potential in vivo: a first human TSPO blocking study. J Cereb Blood Flow Metab. 2014;34(6):989-94. https://doi.org/10.1 038/jcbfm.2014.46.

49. Hagens MHJ, Golla SV, Wijburg MT, Yaqub M, Heijtel D, Steenwijk MD, et al. In vivo assessment of neuroinflammation in progressive multiple sclerosis: a proof of concept study with F-18 DPA714 PET. J Neuroinflammation. 2018;15(1):314. https://doi.org/10.1186/s12974-018-1352-9.

50. Wang Y, Coughlin J, Zhou Y, Ma S, Endres C, Pomper M. A method for personalized brain mapping of neuroinflammation using 11C-DPA-713 PET. J Nucl Med. 2013:54:529.

51. Buchert R, Dirks M, Schütze C, Wilke F, Mamach M, Wirries A-K, et al. Reliable quantification of (18)F-GE-180 PET neuroinflammation studies using an individually scaled population-based input function or late tissue-to-blood ratio. Eur J Nucl Med Mol Imaging. 2020:47(12):2887-900. https://doi.org/10.1007/s00259-020-04810-1.

52. Ye Q, Lyu Z, Yao S, Dong Y, Liu H, Wu J, et al. Direct 4 D Patlak reconstruction in dynamic FDG PET imaging with population-based input function. In: 2018 IEEE Nuclear Science Symposium and Medical Imaging Conference, NSS/MIC 2018 - Proceedings. 2018. p. 1-4.

53. Rissanen E, Tuisku J, Luoto P, Arponen E, Johansson J, Oikonen V, et al. Automated reference region extraction and population-based input function for brain C-11 TMSX PET image analyses. J Cereb Blood Flow Metab. 2015;35(1):157-65. https://doi.org/10.1038/jcbfm.2014.194.

\section{Publisher's Note}

Springer Nature remains neutral with regard to jurisdictional claims in published maps and institutional affiliations.

\section{Submit your manuscript to a SpringerOpen ${ }^{\circ}$ journal and benefit from:}

- Convenient online submission

- Rigorous peer review

- Open access: articles freely available online

- High visibility within the field

- Retaining the copyright to your article

Submit your next manuscript at $\boldsymbol{\nabla}$ springeropen.com 